\title{
A NUMERICAL STUDY OF NON-CAVITATING AND CAVITATING LIQUID FLOW AROUND A HYDROFOIL
}

\author{
François Beux ${ }^{1}$, Maria-Vittoria Salvetti ${ }^{2}$, Alexey Ignatyev ${ }^{3}$, Ding Li $^{4}$, \\ Charles Merkle ${ }^{4}$ And Edoardo Sinibaldi ${ }^{1}$
}

\begin{abstract}
The results of a workshop concerning the numerical simulation of the liquid flow around a hydrofoil in non-cavitating and cavitating conditions are presented. This workshop was part of the conference "Mathematical and Numerical aspects of Low Mach Number Flows" (2004) and was aimed to investigate the capabilities of different compressible flow solvers for the low Mach number regime and for flows in which incompressible and supersonic regions are simultaneously present. Different physical models of cavitating phenomena are also compared. The numerical results are validated against experimental data.
\end{abstract}

Mathematics Subject Classification. 65M99, 76B10, 76G25, $76 \mathrm{~T} 05$.

Numerical workshop, Low Mach Number Flows Conference, June 21-25, 2004, Porquerolles, France.

\section{INTRODUCTION}

The present benchmark problems concern the numerical simulation of the liquid flow around a NACA0015 hydrofoil in non-cavitating and cavitating conditions and were proposed at the conference "Mathematical and Numerical aspects of Low Mach Number Flows", Porquerolles, France, June 21-25 2004 (web site: http:// www-sop.inria.fr/smash/LOMA).

The first proposed test case, i.e. the liquid flow around the hydrofoil in non-cavitating conditions, is a classical benchmark flow of clear interest for aerospace, naval and industrial applications, which is characterized by a very low Mach number. Although there are in the literature numerous examples of numerical simulations of similar problems through incompressible flow solvers, since one of the objectives of the conference was to provide an up-to-date view of the behavior of numerical solvers for compressible flows in the low Mach number regime, the contributors were asked to use a compressible flow numerical solver. A low angle of attack is considered; thus, the possibility of neglecting viscous effects was left to contributors. Finally, the hydrofoil is part of a rectangular wing which is placed in a water tunnel spanning the entire width of the test-section; hence, 3D effects are expected not to be important in the central part of the wing. Thus, contributors were allowed either to simulate the entire tunnel width (3D computation) or to carry out a $2 \mathrm{D}$ simulation of the tunnel central section.

\footnotetext{
Keywords and phrases. Low-Mach number, cavitating flows.

1 Scuola Normale Superiore di Pisa, Italy. fbeux@sns.it; e.sinibaldi@sns.it

2 Dipartimento di Ingegneria Aerospaziale, Università di Pisa, Italy. mv.salvetti@ing.unipi.it

${ }^{3}$ Institute for High Performance Computing and Data Bases, St.Petersburg State Polytechnical University, Russia. iaa@gtn.ru

4 Mechanical Engineering, Purdue University, USA. dli@purdue.edu; merkle@purdue.edu
} 
For validation, a reference numerical solution is provided, obtained by means of incompressible potential flow solvers [1]; pressure experimental data over the hydrofoil are also available [11].

A second test-case was also proposed; it consists in the simulation of the same geometry as previously, but such that a cavitating bubble forms on the leeward side of the hydrofoil. The investigation and prediction of cavitation phenomena is of crucial importance, for instance, in liquid propellant rocket engines and in biomedical, environmental and material processing fields.

From a numerical viewpoint, this test-case is particularly challenging because both incompressible zones (pure liquid) and regions where the flow may easily become highly supersonic (liquid-vapor mixtures) are present in the flow and need to be solved simultaneously. The numerical stiffness of the problem is further increased both by the high liquid-to-vapor density ratio (which, for instance, is of the order of $10^{5}$ for water-vapor mixture at $20^{\circ} \mathrm{C}$ ) and by the strong shock-like discontinuities occurring in the recondensation at the cavity closure. This singular behavior reflects the large variations of the sound speed with the pressure as the flow transitions from a fully-wetted liquid to a two-phase cavitating mixture. Thus, this second test-case is aimed to investigate the capabilities of numerical solvers to simulate flows characterized by the simultaneous presence of nearly incompressible regions together with highly supersonic ones. Although the effects of viscosity and turbulence are not clear, also for this case, possibility was left to the contributors of neglecting viscosity or turbulence. Freedom was also left for the choice of the physical modeling of the cavitation. As previously, either 3D or $2 \mathrm{D}$ simulations could be carried out.

For this test-case, experimental data [11] are available for validation, namely pressure measurements over the hydrofoil and the mean length of the cavitation bubble.

Three contributions to the first problem were received and are presented in the following, and namely contributions by Beux, Salvetti and Sinibaldi (University of Pisa and Scuola Normale Superiore di Pisa, Italy), denoted as SBS in the following, by Li and Merkle, from Purdue University, USA (acronym LM) and by Ignatiev (Ig) from the St. Petersburg State Polytechnical University, Russia. Only SBS and LM contributed to the second benchmark problem.

The paper is organized as follows: the physical modeling adopted in the different contributions is briefly presented in Section 1, while Section 2 describes the main features of the numerical approaches followed by the contributors; for more details we refer to [7-9] for LM, $[13,14]$ for SBS and $[6,10]$ for Ig. In Section 3 the test-case geometry and the flow conditions are described; the results of the different numerical simulations are then presented and analyzed in Section 4 and concluding remarks are finally drawn in Section 5.

\section{Physical MODELS}

\subsection{Non-cavitating flow}

As stated in the introduction, the physical model should be able to consider the fluid compressibility. Conversely, the viscous effects could be neglected and this assumption has been made by all the contributors (SBS, LM and Ig). More specifically, the Euler equations of gas dynamics are used by LM and Ig, while a reduced set of governing equations involving only the mass and momentum balances of the classical Euler equations is considered by SBS. Indeed, in this last case, the flow is assumed to be barotropic, and, thus, the energy equation is decoupled from the rest of the system.

As for constitutive equations, Ig uses the classical equations of state for a perfect gas with $\gamma=1.4$, while the following state law for a weakly compressible barotropic liquid is adopted by SBS:

$$
p=p_{\text {sat }}+\frac{1}{\beta_{s L}} \ln \left(\frac{\rho}{\rho_{L \text { sat }}}\right)
$$

in which $p$ is the pressure, $\rho$ the density, $\beta_{s L}$ the isentropic compressibility assumed to be constant, depending on the specific liquid under consideration, and $p_{\text {sat }}$ and $\rho_{L \text { sat }}$ are the saturation pressure and density respectively; $p_{\text {sat }}$ and $\rho_{L \text { sat }}$ are also determined by the liquid temperature $T_{L}$, which is assumed to be constant and equal to the 
far stream temperature. The following values have been used for the present test cases: $\beta_{s L}=5 \times 10^{-10} \mathrm{~Pa}^{-1}$, $p_{\text {sat }}=2339.953 \mathrm{~Pa}$ and $\rho_{\text {sat }}=997.949 \mathrm{~kg} / \mathrm{m}^{3}$.

On the other hand, LM consider the following state equations:

$$
\left\{\begin{array}{l}
\rho=\rho_{0}+Z p R T \\
H=H_{0}+C_{p}\left(T-T_{\mathrm{ref}}\right)
\end{array}\right.
$$

where $H$ is the flow enthalpy, $R$ the perfect gas constant for water vapor, $\rho_{0}=1000 \mathrm{~kg} / \mathrm{m}^{3}, Z=0.0624$, $H_{0}=112.75 \mathrm{~kJ}, C_{p}=4180 \mathrm{~J} / \mathrm{K}$ and $T_{\text {ref }}=300 \mathrm{~K}$.

\subsection{Cavitating flow}

For the test-case involving cavitation phenomena, SBS still consider an inviscid barotropic flow, and thus, the reduced Euler equations, whereas LM also take into account viscous and turbulent effects. More precisely, LM consider the Reynolds-averaged Navier-Stokes equations with the $\kappa-\omega$ two-equation closure model [18].

The approaches of LM and SBS also differ for the choice of the cavitation model.

The cavitation model adopted by SBS is a homogeneous-flow model explicitly accounting for thermal cavitation effects and for the concentration of the active cavitation nuclei in the liquid [2]. According to this model, the flow behaves isentropically, so that it is possible to use the energy balance of the mixture in order to evaluate the mass interaction term accounting for evaporation/condensation phenomena between the two phases and ultimately derive a constitutive relation linking the density and the pressure of the cavitating mixture. As a result, it can be assumed that the entire flow is barotropic [2]. In non cavitating regions, i.e. for pure liquid $\left(\rho \geq \rho_{\text {sat }}\right)$, the state law defined in equation (1) is adopted, whereas for the cavitating regions, i.e. for $\left(\rho<\rho_{\text {sat }}\right)$, the following equation holds:

$$
\frac{1}{\rho} \frac{\mathrm{d} \rho}{\mathrm{d} p}=\frac{1-\alpha}{p}\left[\beta_{s L}\left(1-\varepsilon_{L}\right) p+\varepsilon_{L} g^{\star}\left(\frac{p_{c}}{p}\right)^{\eta}\right]+\frac{\alpha}{\gamma p}
$$

where $g^{\star}, \eta, p_{c}$ and $\gamma$ are given constants depending only on the working liquid. The volume fraction of the liquid which is in thermal equilibrium with the bubbles is determined by $\varepsilon_{L}\left(0 \leq \varepsilon_{L} \leq 1\right)$. It is a given function of the approximated void fraction $\alpha=1-\rho / \rho_{L \text { sat }}$ and of $\delta_{T} / R$, which is a free parameter accounting for thermal effects and the concentration of the active cavitation nuclei [2]. In the present test-case the following values of the different parameters corresponding to water at $T=25^{\circ} \mathrm{C}$ have been used, and, namely, $g^{\star}=1.67, \eta=0.73$, $\gamma=1.28$ and $p_{c}=2.2089 \times 10^{7} \mathrm{~Pa}$.

The cavitation model chosen by LM is a two-phase flow model in which transport equations are solved for liquid and vapor mass fractions $\left(Y_{l}\right.$ and $Y_{v}$ respectively). The state and caloric equations have the same form for both liquid and vapor, i.e. equations (2) are also used for vapor but with the following values of the parameters: $\rho_{0}=0 \mathrm{~kg} / \mathrm{m}^{3}, Z=1, H_{0}=2796.0 \mathrm{~kJ}, C_{p}=2050.8 \mathrm{~J} / \mathrm{K}$ and $T_{\text {ref }}=400 \mathrm{~K}$. A source term is included in the transport equations to allow for transformation between liquid and vapor phases [3]. The source term $S_{Y}=\dot{m}^{+}+\dot{m}^{-}$is directly obtained from the vapor generation/condensation rates, which are described using the following finite-rate relations:

$$
\dot{m}^{+}=C_{\text {prod }} \frac{\rho Y_{l} \max \left(0, p_{v}-p\right)}{\frac{1}{2} \rho_{\text {in }} U_{\text {in }}^{2} t_{\text {ref }}} \text { and } \quad \dot{m}^{-}=C_{\text {dest }} \frac{\rho Y_{v} \min \left(0, p_{v}-p\right)}{\frac{1}{2} \rho_{\text {in }} U_{\text {in }}^{2} t_{\text {ref }}} .
$$

$C_{\text {prod }}$ and $C_{\text {dest }}$ are empirical constants, and $t_{\text {ref }}$ is a mean flow time scale. $C_{\text {prod }}=10^{5}, C_{\text {dest }}=1$ and $t_{\text {ref }}=U_{\text {in }} / c, U_{\text {in }}$ being the inlet velocity and $c$ the length of the hydrofoil chord; $p_{v}$, the phase-change threshold pressure value, is not set equal to the saturation vapor pressure, but to an ad-hoc fixed value $\left(p_{v}=5372 \mathrm{~Pa}\right)$, in order to approximately take into account turbulence effects on cavitating phenomena. 
TABLE 1. Summary of the different adopted physical models.

\begin{tabular}{|c|c|c|c|}
\hline & $\operatorname{Ig}$ & LM & SBS \\
\hline \multicolumn{4}{|c|}{$\underline{\text { Non-cavitating flows }}$} \\
\hline governing equations & 2D Euler & 2D Euler & reduced 3D Euler \\
\hline constitutive equations & perfect gas & state equations (2) & barotropic law (1) \\
\hline \multicolumn{4}{|c|}{ Cavitating flows } \\
\hline governing equations & & $\begin{array}{l}\text { 2D RANS with } \kappa-\omega \text { model } \\
+ \text { transport equations for liquid and vapor }\end{array}$ & reduced 3D Euler \\
\hline constitutive equations & & $\begin{array}{l}\text { two-phase flow model: } \\
\text { state eqs. (2) for liquid and vapor }\end{array}$ & $\begin{array}{l}\text { homogeneous-flow model: } \\
\text { eqs. (1) and (3) }\end{array}$ \\
\hline
\end{tabular}

The main features of the different physical models are summarized in Table 1.

\section{NumericAl APPROACHES}

\subsection{Spatial discretization and preconditioning}

In all the contributions, the spatial discretization is based on an upwind finite-volume formulation with approximate Riemann solvers. Let us consider, for simplicity, a 1D flow with uniform space and time discretizations, then the semi-discrete formulation can be expressed as follows:

$$
\frac{\partial W_{i}}{\partial t}+\frac{1}{\Delta x}\left(\Phi_{i, i+1}-\Phi_{i-1, i}\right)=0
$$

in which $W_{i}$ is the vector of the unknown variables in the cell $i$ and the numerical fluxes at the cell interfaces may be expressed as:

$$
\Phi_{l, r}=\Phi\left(W_{l}, W_{r}\right)=\frac{F\left(W_{l}\right)+F\left(W_{r}\right)}{2}-\frac{1}{2}\left|\tilde{A}\left(W_{l}, W_{r}\right)\right|\left(W_{r}-W_{l}\right)
$$

in which $F(W)$ is the exact convective flux and $\tilde{A}\left(W_{l}, W_{r}\right)$ is a flux Jacobian with respect to $W$. SBS and Ig use the Roe scheme, i.e. the Jacobian is evaluated using Roe averages (the classical ones defined for the Euler equations with the perfect gas state equation [12] for Ig, modified ones for barotropic flows [4,13] for SBS).

A well-known problem for this kind of formulation is that, dealing with a nearly incompressible flow, the accuracy becomes very poor. Thus, preconditioning must be applied for the low Mach number regime. Following 
[5], a preconditioning modifying only the upwind part of the numerical flux function can be introduced as follows:

$$
\Phi_{l r}^{\text {prec }}=\frac{1}{2}\left(F\left(W_{l}\right)+F\left(W_{r}\right)\right)-\frac{1}{2} P_{l r}^{-1}\left|P_{l r} \tilde{A}_{l r}\right|\left(W_{r}-W_{l}\right) .
$$

Ig starts from equation (6) using primitive variables, i.e. for the $1 \mathrm{D}$ case $U=\left(p, u, \ln \left(p / \rho^{\gamma}\right)\right)^{T}$, and considering as preconditioning matrix the simplest preconditioner of Turkel type $[5,15]$, i.e. $P=\operatorname{diag}\left(\beta^{2}, 1,1\right)$, in which the free parameter $\beta$ is set equal to the typical Mach number of the flow.

SBS use conservative variables, i.e. in a 1D context and with the reduced governing equations $Q=(\rho, \rho u)^{T}$. Nevertheless, the preconditioning matrix is first derived in primitive variables with the same choice as Ig: $P(U)=\operatorname{diag}\left(\beta^{2}, 1\right)$ with $U=(p, u)^{T}$ and $\beta$ is assumed to be proportional to a reference Mach number. Then, returning to the conservative variables by $P(Q)=\frac{\partial Q}{\partial U} P(U) \frac{\partial U}{\partial Q}$ the following expression of the preconditioning matrix in equation (6) is obtained:

$$
P_{l r}=\left(\begin{array}{cc}
\beta^{2} & 0 \\
\tilde{u}_{l r}\left(\beta^{2}-1\right) & 1
\end{array}\right) \quad \text { where } \tilde{u}_{l r} \text { is the value of the Roe average of } u .
$$

Due to the fact that the time derivative has not been modified, the time-consistency is maintained and the method with preconditioning is also applicable to unsteady problems. Nevertheless, this formulation does not cure the problem of inefficiency for low Mach limit, and thus, ad hoc efficient time-advancing schemes must be used (see Sect. 2.2).

Alternatively, starting from governing equations in conservative variables $\left.\left(\text { i.e., } Q=\left(\rho, \rho u, \rho h^{0}-p\right)\right)^{T}\right)$, LM rewrite the equations choosing the primitive variables (i.e., $\left.U=(p, u, T)^{T}\right)$ as the primary dependent variables. In this way, the time derivative is expanded by the Jacobian $\partial Q / \partial U$. Then, to avoid singularities and improve accuracy and convergence in the low Mach-number regime, the critical term $\partial \rho / \partial p$ is replaced by $1 / V_{p}^{2}$, where $V_{p}$ is the preconditioning velocity scale and should be the fluid particle velocity to ensure proper conditioning of the time-derivatives terms $[16,17]$. In this way, the resulting matrix $\Gamma$ remains well conditioned even in the incompressible limit, and the resulting scheme is the following:

$$
\Gamma_{i} \frac{\partial U_{i}}{\partial \tau}+\frac{1}{\Delta x}\left(\Phi_{i, i+1}-\Phi_{i-1, i}\right)=0
$$

in which the numerical fluxes are obtained by equation (6) with $P=\Gamma$ and $\tau$ is a pseudo-time. Indeed, since the time derivative term has been modified only steady solutions can be considered.

\subsection{Time discretization}

As previously pointed out, with a time-consistent preconditioning, an implicit formulation for time advancing is useful, since for the low Mach limit the stability condition for explicit schemes becomes too severe. An implicit approach is also useful with a pseudo-time formulation since it allows the convergence to steady solutions to be accelerated.

Let us consider a fully implicit backward Euler scheme, which can be written in a unified way for both the semi-discretizations in equations (5) or (7), as follows:

$$
C_{i} \frac{\Delta^{n} W_{i}}{\Delta t}+\frac{1}{\Delta x}\left(\Phi_{i, i+1}^{n+1}-\Phi_{i-1, i}^{n+1}\right)=0
$$

where $C_{i}=\Gamma_{i}$ or $I_{d}, \Delta t$ can be the time step or the pseudo-time step, and $\Delta^{n}(\cdot)=(\cdot)^{n+1}-(\cdot)^{n}, n$ denoting the $n$-th temporal iteration level.

A linearization of equation (8) can be obtained by introducing the following approximation:

$$
\Delta^{n} \Phi_{l r} \simeq H_{l r}^{1} \Delta^{n} W_{l}+H_{l r}^{2} \Delta^{n} W_{r} .
$$


In this way, an implicit linearized scheme in delta form is obtained by LM (see [9]) and SBS (see [13]). SBS uses a linearization which exploits algebraic properties of the Roe numerical fluxes [13]. As a result, the approximation is also valid for inhomogeneous fluxes as in the barotropic case.

The resulting implicit linearized schemes can be expressed as follows:

- LM formulation: $\Gamma_{i} \frac{\Delta^{n} U_{i}}{\Delta \tau}+\mathcal{B}_{\mathrm{LM}} \frac{\Delta^{n} U_{i}}{\Delta x}=-\frac{\left[\Phi_{U}\right]_{i, i+1}^{n}-\left[\Phi_{U}\right]_{i-1, i}^{n}}{\Delta x}$,

- SBS formulation: $\frac{\Delta^{n} Q_{i}}{\Delta t}+\mathcal{B}_{\mathrm{SBS}} \frac{\Delta^{n} Q_{i}}{\Delta x}=-\frac{\left[\Phi_{Q}\right]_{i, i+1}^{n}-\left[\Phi_{Q}\right]_{i-1, i}^{n}}{\Delta x}$.

The matrices $\mathcal{B}_{\mathrm{LM}}$ and $\mathcal{B}_{\mathrm{SBS}}$ come from the respective linearization of the spatial first-order discrete operator including preconditioning. In order to avoid a too expensive matrix inversion, when the basic numerical treatment of fluxes is modified to obtain higher order accuracy in space, this is done only on the right-hand side of the previous equations, i.e. in the computation of explicit numerical fluxes. SBS only uses first-order accuracy, i.e. exactly equation (6) for the numerical fluxes, while LM consider a second-order spatial scheme.

Alternatively, Ig uses a preconditioned dual-time implicit procedure [10]: to cure the inefficiency for low Mach limit, the time derivative has been multiplied by a preconditioning matrix, but in order to save time-depended consistency, it has been done only for an artificial time. Thus, instead of equation (5), the following semi-discrete formulation is considered:

$$
P^{-1} \frac{\partial W_{i}}{\partial \tau}+\frac{\partial W_{i}}{\partial t}+\frac{1}{\Delta x}\left(\Phi_{i, i+1}-\Phi_{i-1, i}\right)=0
$$

in which the spatial discretization is unchanged and $P$ corresponds to the simplest Turkel preconditioner previously introduced.

Then, the artificial time derivative is discretized with a first-order finite difference while a second-order BDF scheme is used for the physical time derivative. More precisely, the following scheme in delta form is obtained:

$$
P^{-1} \frac{\Delta^{k} U_{i}}{\Delta \tau}+\frac{3 \Delta^{k} U_{i}}{2 \Delta t}+\mathcal{B}_{I g} \frac{\Delta^{k} U_{i}}{\Delta x}=-\Omega_{i}^{k}\left(\frac{3 Q_{i}^{k}-4 Q_{i}^{n}+Q_{i}^{n-1}}{2 \Delta t}+\frac{\left[\Phi_{Q}\right]_{i+1 / 2}^{k}-\left[\Phi_{Q}\right]_{i-1 / 2}^{k}}{\Delta x}\right),
$$

where $\Omega=\partial U / \partial Q$ and $\mathcal{B}_{I g}$ comes from the linearization of the spatial first-order discrete operator including preconditioning. The calculation of the explicit fluxes $\left[\Phi_{Q}\right]_{i \pm 1 / 2}^{k}$ is carried out by a 5 th-order WENO scheme [6].

A factored Newton-type method is constructed by defining a sequence of approximation $\Delta^{k} U$, between two time steps $n$ and $n+1$, where $k$ stands for the Newton sub-iterations. After each Newton cycle, the solution update in primitive variables, $\Delta U$, is converted into conservative ones by multiplying it by $\Omega^{-1}$. This scheme shows good convergence and allows large $C F L_{u+c}$ numbers $\left(10^{2}\right.$ to $\left.10^{4}\right)$ to be used.

The previously described characteristics of the different numerical approaches are summarized in Table 2 .

\section{DESCRIPTION OF THE TEST-CASES}

In the present benchmark, the flow over a NACA0015 hydrofoil is considered. The hydrofoil is mounted in a water tunnel at $4^{\circ}$ angle of attack and spans the entire width of the rectangular test chamber section, which is equal to $0.7 c, c$ being the hydrofoil chord length, equal to $115 \mathrm{~mm}$ (see Fig. 1). This configuration has been considered in an experimental study [11], for which the pressure coefficient distribution on the symmetry plane of the hydrofoil is available. Thus, the symmetry plane of the 3D computational domain can be considered as the computational domain for 2D simulations (Fig. 2). The dimensions of the computational domain are shown in Figure 2.

The working liquid is water at $T=25{ }^{\circ} \mathrm{C}$. Both cavitating and non-cavitating flows can be considered by varying the inlet conditions, which are described in Table 3. For the cavitating case, the cavitation number, defined as $s=\frac{p_{\text {in }}-p_{\mathrm{sat}}}{\frac{1}{2} \rho_{\mathrm{in}} U_{\mathrm{in}}^{2}}$, is equal to 1.52 . 
TABLE 2. Main characteristics of the different numerical approaches.

\begin{tabular}{|c|c|c|c|}
\hline & $\operatorname{Ig}$ & LM & SBS \\
\hline $\begin{array}{c}\text { spatial } \\
\text { discretization }\end{array}$ & $\begin{array}{l}\text { finite volume } \\
\text { for structured grids } \\
\text { upwind schemes }\end{array}$ & $\begin{array}{l}\text { finite volume } \\
\text { for unstructured grids } \\
\text { upwind schemes }\end{array}$ & $\begin{array}{l}\text { finite volume } \\
\text { for unstructured grids } \\
\text { upwind schemes }\end{array}$ \\
\hline spatial accuracy & WENO 5 th-order & second-order & first-order \\
\hline preconditioning & Turkel type & regularized $\partial Q / \partial U$ & $\begin{array}{c}\text { Turkel type } \\
\text { (conservative form) }\end{array}$ \\
\hline $\begin{array}{c}\text { time } \\
\text { discretization }\end{array}$ & $\begin{array}{l}\text { artificial and physical } \\
\text { time derivatives: } \\
\text { preconditioned dual-time } \\
\text { implicit formulation }\end{array}$ & $\begin{array}{l}\text { pseudo-time (steady solutions) } \\
\text { implicit linearized } \\
\text { scheme in delta form }\end{array}$ & $\begin{array}{c}\text { time-consistent } \\
\text { implicit linearized } \\
\text { scheme in delta form }\end{array}$ \\
\hline time accuracy & second-order BDF & first-order & first-order \\
\hline variables & primitive - conservative & primitive & conservative \\
\hline
\end{tabular}

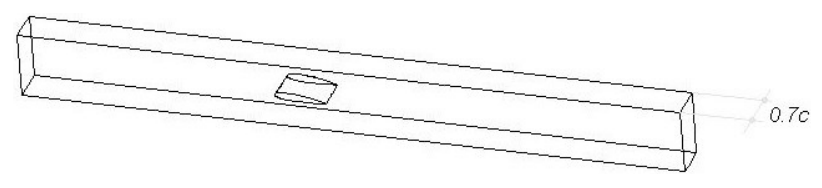

Figure 1. Test-case geometry.

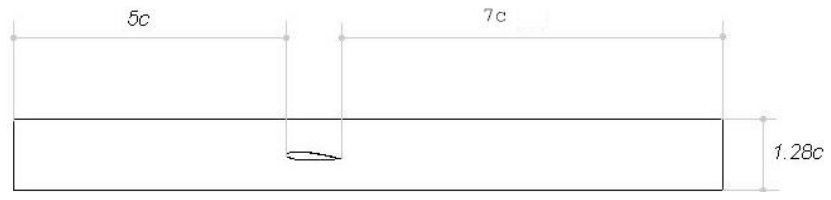

FiguRE 2. Symmetry plane of the water tunnel and 2D computational domain.

\section{Results AND Discussion}

\subsection{Non-cavitating flow}

For this first problem, Ig and LM carried out 2D simulations, by discretizing the symmetry section of the water tunnel (see Fig. 2). Ig used a multi-block structured grid having 7952 cells (129 nodes on the hydrofoil), while LM employed a hybrid structured/unstructured grid, structured near the hydrofoil and having 38371 elements (315 nodes on the hydrofoil). SBS carried out two 3D simulations; in the first one, the entire 3D computational domain is discretized by an unstructured grid having 180000 tetrahedra, while, in the second one, only a slice of $0.1 c$ width is considered and discretized by 88400 tetrahedra (151 nodes on the hydrofoil). The main features of the different simulations are summarized in Table 4. 
TABLE 3. The inlet experimental conditions.

\begin{tabular}{|c|c||c|c|}
\hline \multicolumn{2}{|c||}{} & non-cavitating flow & cavitating flow \\
\hline \hline $\begin{array}{c}\text { Temperature } \\
\text { in Kelvin }\end{array}$ & $T_{\text {in }}$ & 298 & 298 \\
\hline $\begin{array}{c}\text { velocity } \\
\text { in } \mathrm{m} / \mathrm{s}\end{array}$ & $U_{\text {in }}$ & 3.11 & 3.41 \\
\hline $\begin{array}{c}\text { pressure } \\
\text { in bar }\end{array}$ & $p_{\text {in }}$ & 0.59 & 0.12 \\
\hline $\begin{array}{c}\text { viscosity } \\
\text { in }(\mathrm{N} \mathrm{s}) / \mathrm{m}^{2}\end{array}$ & $\mu_{\text {in }}$ & 0.00089 & 0.00089 \\
\hline $\begin{array}{c}\text { density } \\
\text { in } \mathrm{kg} / \mathrm{m}^{3}\end{array}$ & $\rho_{\text {in }}$ & 1000 & 1000 \\
\hline Mach number & $M_{\text {in }}$ & 0.0021 & 0.0023 \\
\hline
\end{tabular}

TABLE 4. Main grid characteristics in the different contributions.

\begin{tabular}{|c|c|c|c|}
\hline & $\operatorname{Ig}$ & LM & SBS \\
\hline \multicolumn{4}{|c|}{ Non-cavitating flows } \\
\hline type of grids & $\begin{array}{l}\text { 2D multi-block } \\
\text { structured }\end{array}$ & $\begin{array}{c}\text { hybrid } 2 \mathrm{D} \\
\text { unstructured/structured }\end{array}$ & $\begin{array}{c}\text { tetrahedrical 3D } \\
\text { unstructured }\end{array}$ \\
\hline $\mathrm{n}^{o}$ of elements & 7952 & 38371 & $\begin{array}{l}180000 \text { (3D domain) } \\
88400 \text { (slice of } 0.1 \mathrm{c} \text { ) }\end{array}$ \\
\hline $\begin{array}{l}\mathrm{n}^{o} \text { nodes on } \\
\text { the hydrofoil }\end{array}$ & 129 & 315 & $\begin{array}{c}151 \\
\text { (symmetry section) }\end{array}$ \\
\hline \multicolumn{4}{|c|}{ Cavitating flows } \\
\hline type of grids & & $\begin{array}{c}\text { hybrid 2D } \\
\text { unstructured/structured }\end{array}$ & $\begin{array}{c}\text { tetrahedrical 3D } \\
\text { unstructured }\end{array}$ \\
\hline $\mathrm{n}^{o}$ of elements & & 108000 & 88400 (slice of $0.1 \mathrm{c}$ ) \\
\hline $\begin{array}{l}\mathrm{n}^{o} \text { nodes on } \\
\text { the hydrofoil }\end{array}$ & & 540 & $\begin{array}{c}151 \\
\text { (symmetry section) }\end{array}$ \\
\hline
\end{tabular}

In all the computations, since the assumption of inviscid flow was made, free-slip boundary conditions were used at the hydrofoil and at the lateral walls; inflow conditions were specified according to Table 3.

Figure 3 shows the pressure coefficient distribution at the hydrofoil obtained in the different simulations. The pressure coefficient is defined as: $C_{p}=\frac{p-p_{\text {in }}}{1 / 2 \rho_{\text {in }} U_{\text {in }}^{2}}$. Since, as expected, $3 \mathrm{D}$ effects are not critical in the present case and no significant differences are observed between the two computations carried out by SBS (see, 


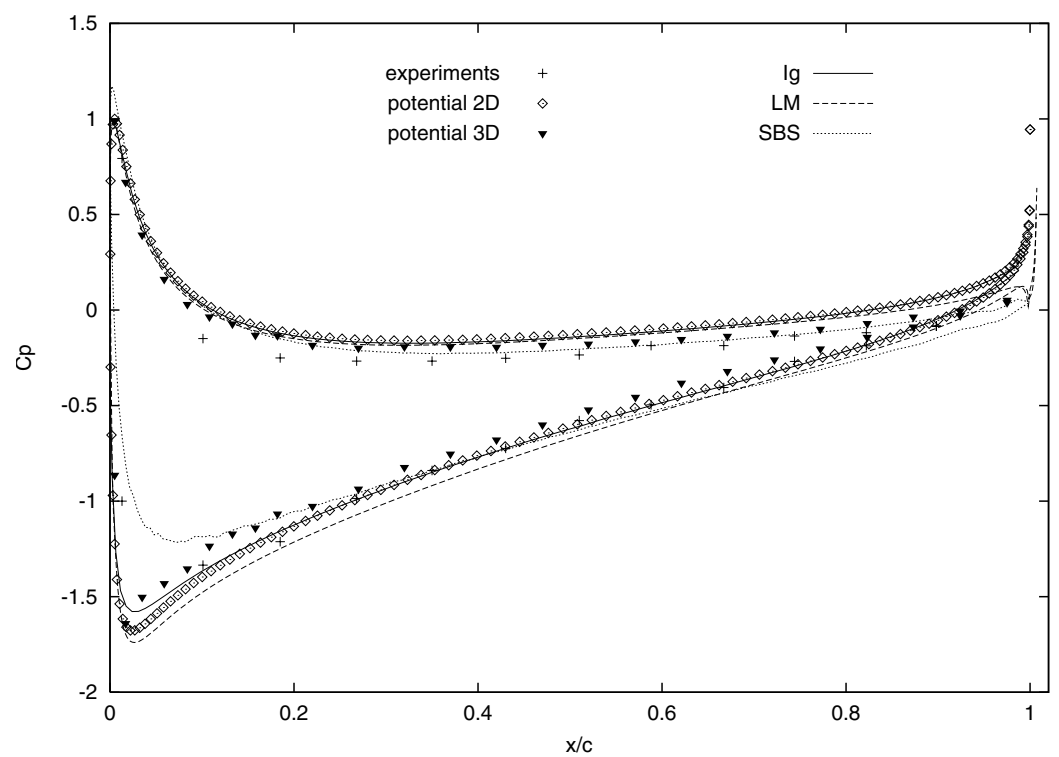

FIGURE 3. Non-cavitating flow: pressure coefficient distribution on the hydrofoil.

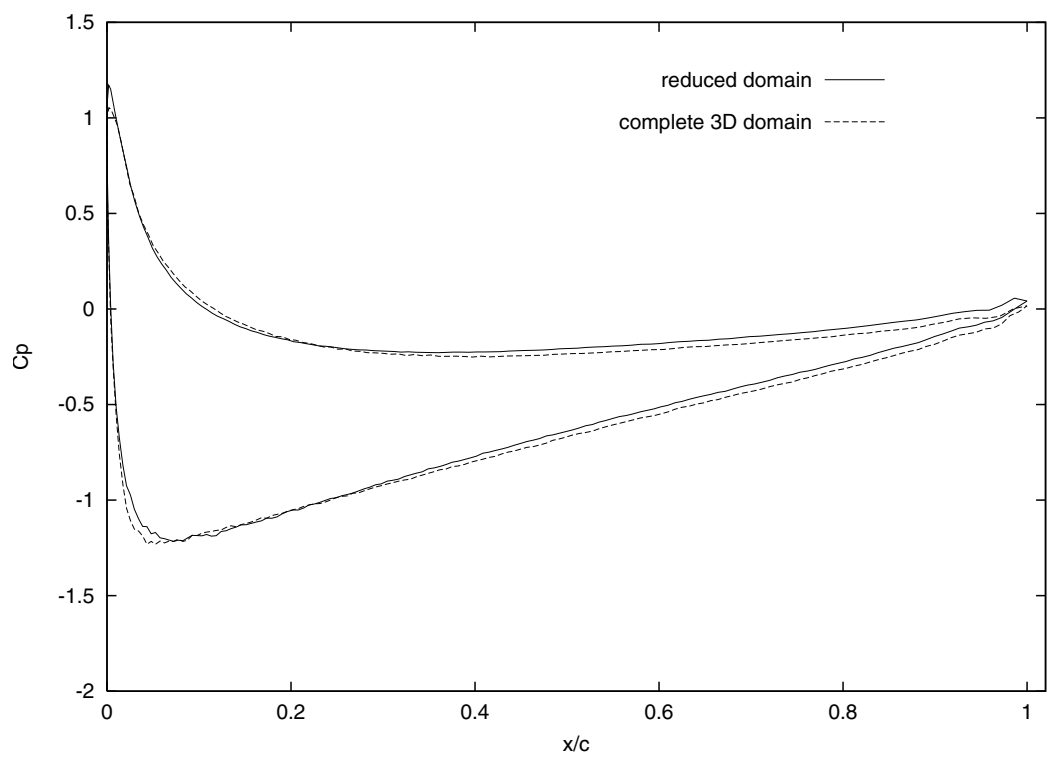

FiguRE 4. Non-cavitating flow: pressure coefficient distribution on the hydrofoil obtained in SBS simulations with different computational domains.

e.g., Fig. 4), only the simulation on the domain slice is shown in Figure 3 for SBS. Experimental data are also reported in Figure 3 together with the solutions obtained by a $2 \mathrm{D}$ and a $3 \mathrm{D}$ potential solver. On the windward side, fairly good agreement is observed between all the simulations and the reference data, except near the trailing edge where LM and Ig results become more far from the experimental data. This is due to the fact that these simulations are $2 \mathrm{D}$, and, indeed, a similar behavior is observed in the $2 \mathrm{D}$ potential flow 


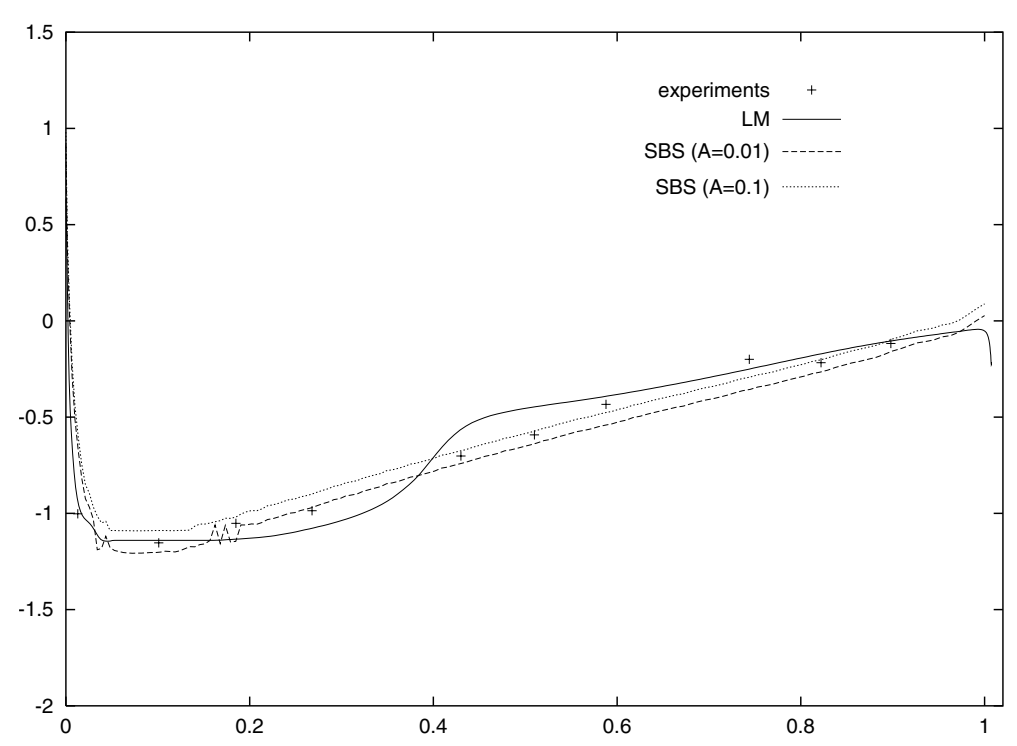

FiguRE 5. Cavitating flow: pressure coefficient distribution on the leeward side of the hydrofoil.

solution. Consistently, the best agreement with the experimental data near the trailing edge is obtained in the 3D simulation by SBS. Conversely, on the leeward side, the SBS results show a significant underestimate of the suction peak near the leading edge. This is due to the low order of spatial accuracy of the numerical method (1st order) together with the moderate grid resolution near the hydrofoil. Indeed, the simulation by SBS has a much lower resolution near on the hydrofoil than the one by LM, in which a second-order spatial accuracy is employed (see Tab. 4); Ig has a resolution comparable to SBS, but the numerical method is 5th-order accurate in space.

\subsection{Cavitating flow}

For this second problem, SBS used the same reduced computational domain and the same grid as in the previous case, while a more refined hybrid mesh having approximately 108000 elements has been employed by LM. SBS carried out two different simulations with two different values of the free-parameter in the cavitation model, $A=\delta_{T} / R(A=0.1$ and $A=0.01)$.

The $C_{p}$ distribution on the leeward side of the hydrofoil obtained in the different simulations is compared with the experimental data in Figure 5. The presence of a cavitation bubble forming near the leading edge is clearly detectable in all the simulations (it corresponds to the plateau in the $C_{p}$ distribution). However, significant differences are observed between the results obtained in the various simulations. The LM $C_{p}$ distribution shows a larger plateau than those of the $S B S$ simulations, indicating a larger width of the cavitating bubble; this behavior well agrees with the experimental data near the leading edge, but significant discrepancies are observed at the edge of the cavitating bubble $(x / c \simeq 0.4)$. Although the presence of some wiggles, the best overall agreement with the experimental $C_{p}$ distribution is obtained in the SBS simulation with $\delta_{T} / R=0.01$. Nevertheless, the LM simulation gives a more accurate prediction of the mean length of the cavitation bubble; indeed in the experiments the length has been estimated to oscillate in time between $0.4 c$ to $0.46 c$ and in the LM simulation is roughly equal to $0.4 c$ (see, for instance, the iso-contours of the vapor volume fraction in Fig. 6 ). Conversely, the length obtained in the SBS computation with $\delta_{T} / R=0.01$ is approximately $0.2 c$ and in the other simulation is lower, as can be seen in Figure 8, in which the iso-contours of the cavitation number are shown.

In this second test case, beside the differences in the spatial accuracy of the numerical method and in the grid resolution, already highlighted for the previous problem, the physical model adopted to describe the cavitation 


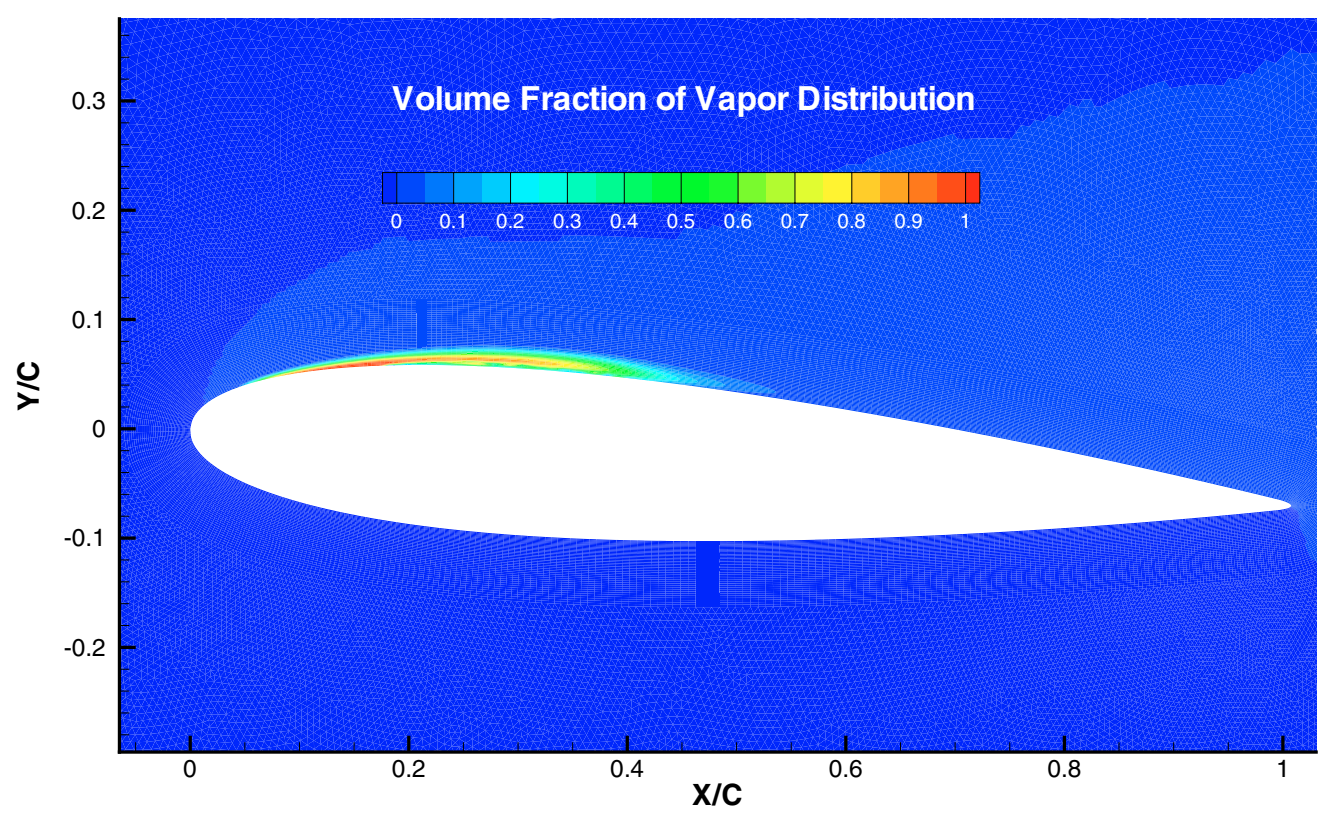

FIGURE 6. Cavitating flow: iso-contours of vapor volume fraction in the LM simulation.

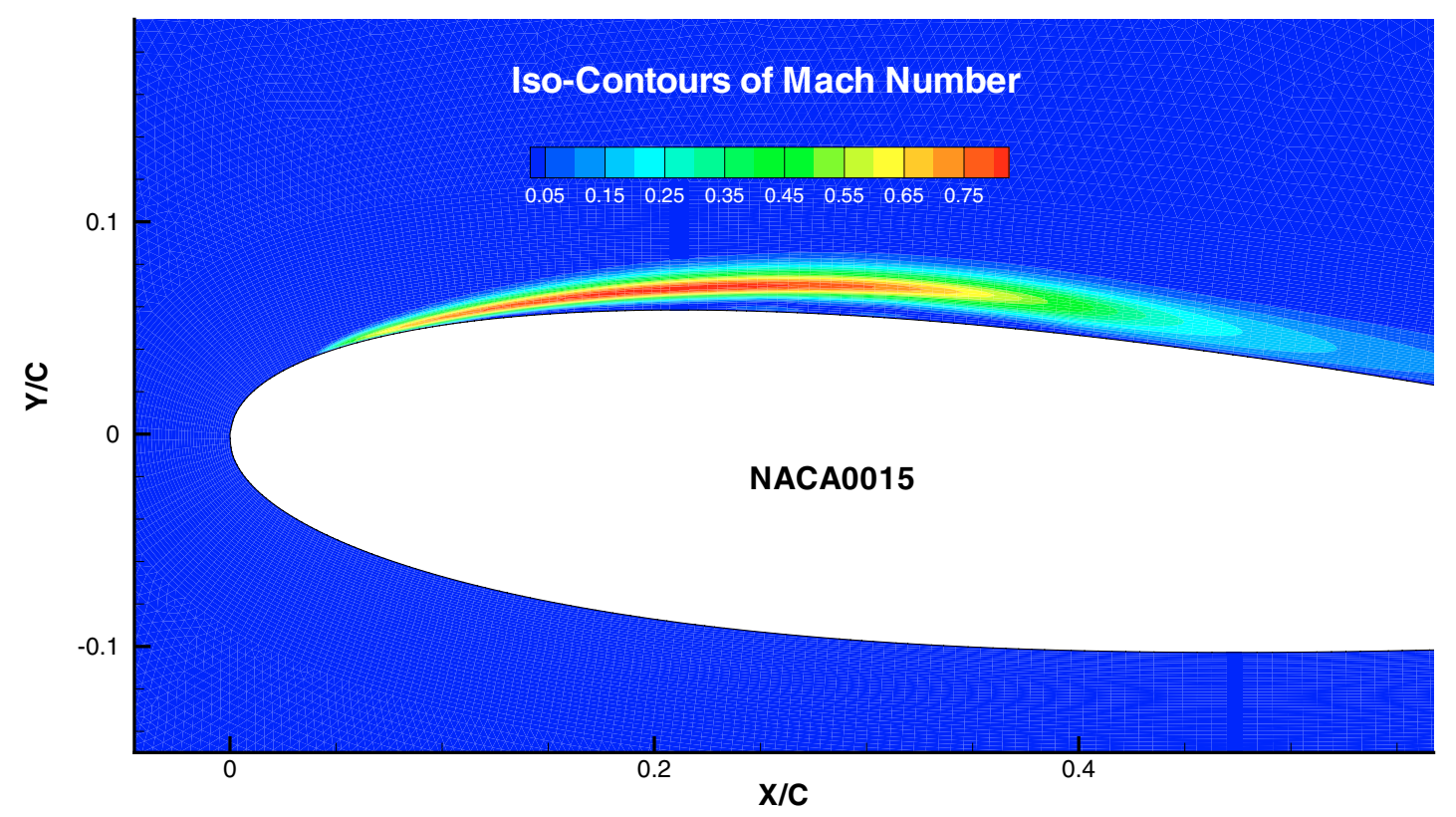

FiguRE 7. Cavitating flow: iso-contours of Mach number in the LM simulation.

phenomena clearly has a great influence on the numerical results. The cavitation models used by LM and SBS have a largely different behavior, as can be seen, for instance, from the iso-contours of the Mach number obtained in the different simulations and shown in Figures 7 and 9. Indeed, in the LM computation the maximum value of the Mach number reached in the cavitating region is of the order of 1, while in the SBS ones is much larger (up to 30 in the simulation with $\delta_{T} / R=0.1$ ). Furthermore, as previously stated, LM take into account flow 
(a)

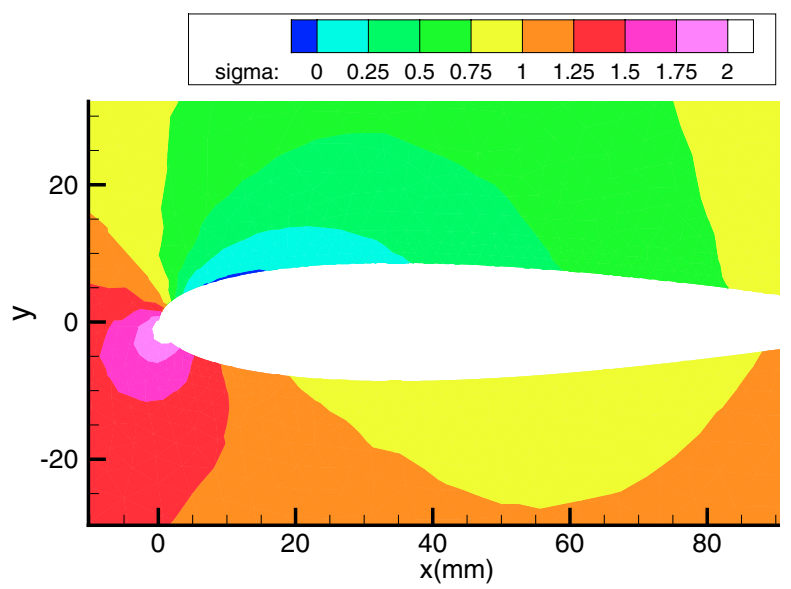

(b)

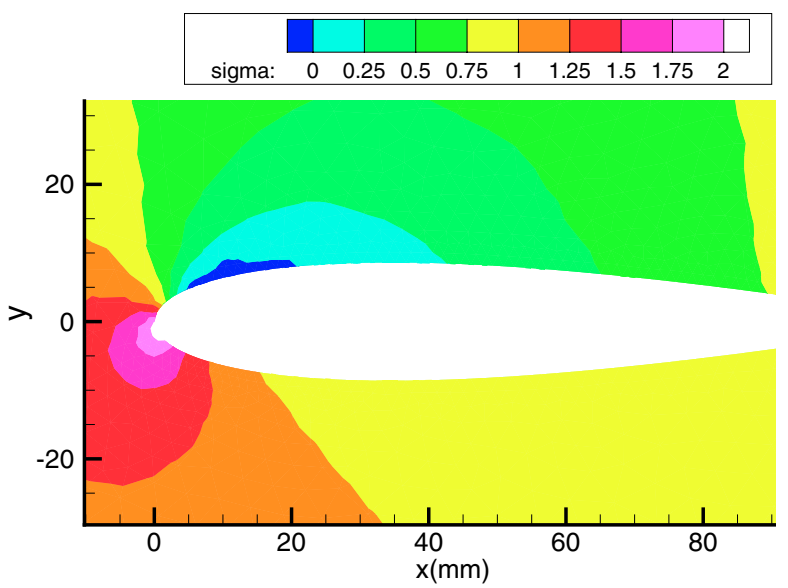

FiguRe 8 . Cavitating flow: iso-contours of cavitation number in the symmetry plane for the SBS simulations; (a) $\delta_{T} / R=0.1$; (b) $\delta_{T} / R=0.01$.

viscosity and turbulence effects, while SBS still consider the flow as inviscid. In spite of these differences, both Figures 7 and 9 clearly illustrate the numerical stiffness of the present problem, characterized by the simultaneous presence of extremely low Mach number regions together with supersonic or highly supersonic zones.

\section{Concluding Remarks}

The results of a numerical workshop, held at the conference "Mathematical and Numerical aspects of Low Mach Number Flows" (2004) and concerning the simulation of the flow around a hydrofoil mounted in a water tunnel, have been presented.

The first test-case consisted in the simulation of the flow at a very low Mach number $\left(M_{\text {in }}=0.002\right)$ in noncavitating conditions and was aimed to compare the behavior of different numerical solvers of compressible flows in the low Mach number regime. Three different contributions have been presented (Ig, LM and SBS). In all the contributions, a finite volume method is used for spatial discretization and the first-order basic component of the computation of numerical fluxes is an approximate Riemann solver. In all cases, preconditioning for low Mach number is applied; SBS and Ig use a Turkel type preconditioning in a time-consistent manner, i.e. only applied to the upwind part of numerical fluxes. In LM preconditioning is obtained by solving the equations 
(a)
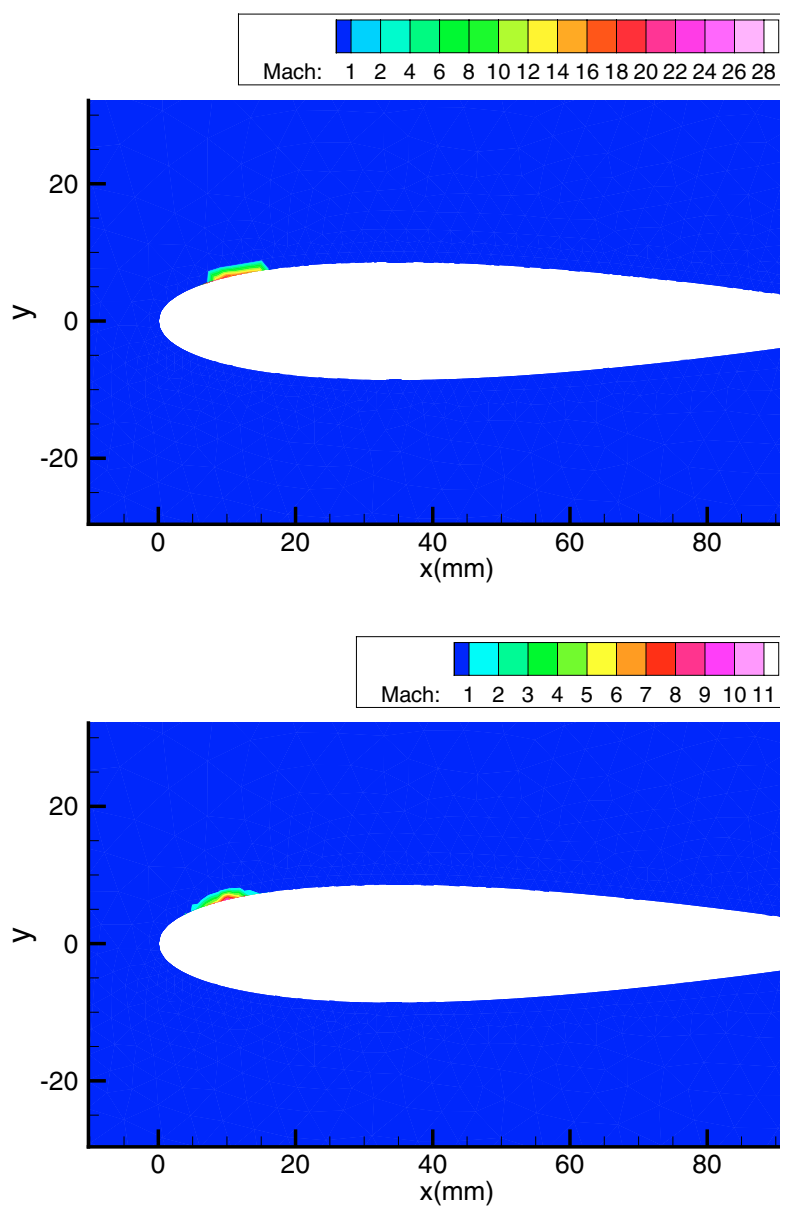

Figure 9. Cavitating flow: iso-contours of Mach number in the symmetry plane for the SBS simulations; (a) $\delta_{T} / R=0.1 ;$ (b) $\delta_{T} / R=0.01$.

in primitive variables; thus, the time-derivative term is pre-multiplied by the Jacobian of the conservative to primitive change of variables, which is properly modified to avoid ill conditioning in the low Mach number limit. The basic method for computing numerical fluxes is then modified in Ig and LM to obtain 5th-order and second-order spatial accuracy respectively; the method by SBS is only first-order accurate in space.

In all the contributions, an ad-hoc efficient implicit time-advancing algorithm is used to overcome the convergence problems typical of the low Mach number limit, namely an implicit linearized scheme in delta form for LM and SBS and dual time stepping for Ig.

The results obtained for this first test case are consistent with the accuracy of the numerical methods and with the employed grid resolutions. This shows that the adopted methods of preconditioning are indeed effective to solve accuracy problems in the low Mach number limit.

The second test-case consisted in the simulation of the flow in the same geometry as previously, but for inflow conditions leading to the formation of a cavitating bubble on the leeward side of the hydrofoil. This problem is more challenging than the previous one both from a numerical viewpoint, since it is characterized by the simultaneous presence of low Mach number and supersonic regions, and from a physical point of view, since the physical modeling of cavitation phenomena is complex and far form being assessed. 
LM and SBS contributed to this test case using the same numerical methods as in the previous one. Conversely different cavitation models were adopted; namely, LM simulated a two-phase flow by numerically discretizing transport equations for liquid and vapor, including source terms to account for vapor generation/condensation. On the other hand, SBS used a homogeneous flow model, leading to different barotropic state equations for non-cavitating and cavitating regions.

Both approaches are able to yield the formation of a cavitating bubble on the leeward side of the hydrofoil and the global agreement with the experimental data is fairly good. However, some discrepancies with the experiments are present and significant differences between the numerical solutions are observed, which can not be only explained by the differences in accuracy and grid resolution. Indeed, for this test case, it seems that the physical model of cavitation phenomena plays a crucial role in the accuracy of the results. Further investigations are clearly needed on this topic, but they are beyond the scope of the numerical workshop, which was to investigate whether ad-hoc designed numerical solvers are able to deal with the numerical stiffness of such problems.

Acknowledgements. The work of François Beux, Maria-Vittoria Salvetti and Edoardo Sinibaldi has been partially supported by a grant from the PACA region to promote French-Italian scientific collaboration and by the Italian Space Agency (ASI) under the research project FAST2, as sub-contractor of CIRA (CR 10/2002).

\section{REFERENCES}

[1] A. Baston, M. Lucchesini, L. Manfriani, L. Polito and G. Lombardi, Evaluation of pressure distributions on an aircraft by two different panel methods and comparison with experimental measurements, in 15th Int. Council of the Aeronautical Sciences Congress, London (1986) 618-628.

[2] L. d'Agostino, E. Rapposelli, C. Pascarella and A. Ciucci, A Modified Bubbly Isenthalpic Model for Numerical Simulation of Cavitating Flows, in 37th AIAA/ASME/SAE/ASEE Joint Propulsion Conference, Salt Lake City, UT, USA, July 8-11 (2001).

[3] M. Deshpande, J. Feng and C. Merkle, Navier-Stokes analysis of 2-D cavity flows. ASME Cavitation and Multiphase Flow Forum, FED-153 (1993) 149-155.

[4] P. Glaister, A Riemann Solver for barotropic flow. J. Comput. Phys. 93 (1991) 477-480.

[5] H. Guillard and C. Viozat, On the behaviour of upwind schemes in the low Mach number limit. Comput. Fluids 28 (1999) 63-86.

[6] G. Jiang and C. Shu, Efficient implementation of weighted ENO schemes. J. Comput. Phys. 126 (1996) 202-228.

[7] D. Li and C. Merkle, Application of a general structured-unstructured solver to flows of arbitrary fluids, in First International Conference on Computational Fluid Dynamics, Kyoto, Japan, July 10-14 (2000).

[8] D. Li, G. Xia and C. Merkle, Analysis of real fluid flows in converging diverging nozzles. AIAA Paper $2003-4132$ (2003), submitted.

[9] D. Li, S. Venkateswaran, K. Fakhari and C. Merkle, Convergence assessment of general fluid equations on unstructured hybrid grids. AIAA Paper 2001-2557 (2001).

[10] S. Pandya, S. Venkateswaran and T. Pulliam, Implementation of preconditioned dual-time procedures in OVERFLOW. AIAA Paper 2003-0072 (2003).

[11] E. Rapposelli, A. Cervone, C. Bramanti and L. d'Agostino, Thermal cavitation experiments on a NACA 0015 hydrofoil, in Proc. of FEDSM'03 4th ASME/JSME Joint Fluids Engineering conference, Honolulu, Hawaii, USA, July 6-11 (2003).

[12] P.L. Roe, Approximate Riemann solvers, parameters vectors, and difference schemes. J. Comput. Phys. 43 (1981) 357-372.

[13] E. Sinibaldi, F. Beux and M.V. Salvetti, A preconditioned implicit Roe's scheme for barotropic flows: towards simulation of cavitation phenomena. INRIA research report No. 4891 (2003).

[14] E. Sinibaldi, F. Beux and M.V. Salvetti, A preconditioned compressible flow solver for numerical simulation of 3D cavitation phenomena, ECCOMAS 2004, 4th European Congress on Computational Methods in Applied Sciences and Engineering, Jyväskylä, Finland, July 24-28 (2004).

[15] E. Turkel, Preconditioned methods for solving the incompressible and low speed compressible equations. J. Comput. Phys. 72 (1987) $277-298$.

[16] S. Venkateswaran and C. Merkle, Analysis of preconditioning methods for Euler and Navier-Stokes equations. 30th VKI computational fluid dynamics lecture series (1999).

[17] S. Venkateswaran, D. Li and C. Merkle, Influence of stagnation regions on preconditined solutions at low speeds. AIAA Paper 2003-0435 (2003).

[18] D.C. Wilcox, Turbulence Modeling for CFD. DCW Industries, Inc., ISBN 0-9636051-5-1 (1998). 